\title{
Large Deviations and the Distribution of Pre-images of Rational Maps
}

\section{Mark Pollicott, Richard Sharp}

Department of Mathematics, University of Manchester, Oxford Road, Manchester M13 9PL, United Kingdom

Received: 1 February 1996/Accepted: 8 May 1996

\begin{abstract}
In this article we prove a large deviation result for the pre-images of a point in the Julia set of a rational mapping of the Riemann sphere. As a corollary, we deduce a convergence result for certain weighted averages of orbital measures, generalizing a result of Lyubich.
\end{abstract}

\section{Introduction}

Let $\hat{\mathbb{C}}$ denote the Riemann sphere and let $T: \hat{\mathbb{C}} \rightarrow \hat{\mathbb{C}}$ be a rational map of degree $d \geqq 2$, say. Every point has $d$ pre-images (counted according to their multiplicities). There is a well-known result of Lyubich which shows that for a point $x \in \mathbb{J}$ in the Julia set an evenly distributed weight on the set of $d^{n}$ pre-images

$$
S_{n}(x)=\left\{y \in \hat{\mathbb{C}}: T^{n} y=x\right\}
$$

converges (in the weak ${ }^{*}$ topology) to a measure $\mu_{0}$ as $n \rightarrow+\infty[4,8]$. The measure $\mu_{0}$ is precisely the unique measure of maximal entropy for the map $T[2,5]$.

Since $T: \mathbb{J} \rightarrow \mathbb{J}$ is a continuous map on a compact metric space we can define the pressure of a continuous function $f: \mathbb{J} \rightarrow \mathbb{R}$ by

$$
P(f)=\sup \left\{h(v)+\int f d v: v \text { is a } T \text {-invariant probability }\right\},
$$

where $h(v)$ denotes the entropy of $T$ with respect to $v$. An equilibrium state for $f$ is a $T$-invariant probability $\mu$ realising this supremum.

Let $\mathscr{M}$ denote the set of all probability measures on $\mathbb{J}$. We shall show the following stronger "large deviation" result on the pre-images of a point $x \in \mathrm{J}$.

Theorem 1. Let $f: \mathbb{J} \rightarrow \mathbb{R}$ be a Hölder continuous function such that $P(f)>$ $\sup f$ and let $\mu$ be the unique equilibrium state for $f$. Let $x \in \mathbb{J}$. Then for any weak $^{*}$ open neighbourhood $\mathscr{U} \subset \mathscr{M}$ of $\mu$ we have that the weighted proportion of 
the measures

$$
\mu_{y, n}=\frac{1}{n}\left(\delta_{y}+\delta_{T y}+\cdots+\delta_{T^{n-1} y}\right) \notin \mathscr{U}
$$

tends to zero exponentially fast, in the sense that there exists $C>0$ and $0<\eta<1$ such that

$$
\frac{\sum_{\substack{T^{n} y=x \\ \mu_{y, n} \notin \mathscr{U}}} e^{f^{n}(y)}}{\sum_{T^{n} y=x} e^{f^{n}(y)}} \leqq C \eta^{n}, \quad \text { for } n \geqq 0,
$$

where we denote $f^{n}(y)=f(y)+f(T y)+\cdots+f\left(T^{n-1} y\right)$.

Remark. The condition $P(f)>\sup f$ was first introduced by Urbanski in [9]. In the present article, as well as being required as a hypothesis for Lemmas 2 and 3, it is also used to give the lower bound required in establishing Lemma 6.

In the special case that $f=0$ then $\mu$ becomes the measure of maximal entropy $\mu_{0}$ for $T: \mathbb{J} \rightarrow \mathbb{J}$. The theorem then reduces to the following.

Corollary 1. Let $x \in \mathbb{J}$. For any weak* open neighborhood $\mathscr{U} \subset \mathscr{M}$ of $\mu_{0}$ the proportion of the points $y \in S_{n}(x)$ such that

$$
\mu_{y, n}=\frac{1}{n}\left(\delta_{y}+\delta_{T y}+\cdots+\delta_{T^{n-1} y}\right) \notin \mathscr{U}
$$

tends to zero exponentially fast, i.e., there exists $C>0$ and $0<\eta<1$ such that

$$
\frac{1}{d^{n}} \#\left\{y \in S_{n}(x): \mu_{y, n} \notin \mathscr{U}\right\} \leqq C \eta^{n}, \quad \text { for } n \geqq 0 \text {. }
$$

A second corollary to the theorem is given by the following convergence result.

Corollary 2. Let $x \in \mathbb{J}$. Let $f: \mathbb{J} \rightarrow \mathbb{R}$ be a Hölder continuous function such that $P(f)>\sup f$ and let $\mu$ be the unique equilibrium state for $f$. Then the averages

$$
\frac{\sum_{T^{n} y=x} e^{f^{n}(y)} \mu_{y, n}}{\sum_{T^{n} y=x} e^{f^{n}(y)}}
$$

converges to $\mu$ in the weak* topology as $n \rightarrow+\infty$.

These two corollaries provide two different generalizations of the following wellknown result of Lyubich.

Lyubich's Theorem $([4,8,2])$. Let $x \in \mathbb{J}$. Then the averages

$$
\frac{1}{d^{n}} \sum_{T^{n} y=x} \mu_{y, n}
$$

converge to $\mu_{0}$ in the weak ${ }^{*}$ topology as $n \rightarrow+\infty$.

Remark. Strictly speaking, Lyubich established that the averages $\frac{1}{d^{n}} \sum_{T^{n} y=x} \mu_{y, n}$ converge to a non-atomic $T$-invariant probability measure supported on $\mathbb{J}$. This measure was subsequently shown to maximise the entropy in [2] and it was shown that there is a unique measure of maximal entropy in [5]. Whereas Lyubich's 
Theorem can be established using normality of sequences of functions and the Montel-Carathéodory theorem [8], these stronger results (Corollaries 1 and 2) seem to require a different argument.

Previous applications of large deviation ideas to rational maps include the result of Lopes dealing with almost everywhere convergence of Birkhoff averages [3]. (Lopes restricted himself to the case of hyperbolic Julia sets but, as Przytycki observed [7] this is unnecessary provided we assume $P(f)>\sup f$.)

\section{Some Properties of Rational Maps}

In this section we shall recall some of the basic properties of rational maps which we shall need later. Let $T: \hat{\mathbb{C}} \rightarrow \hat{\mathbb{C}}$ be a rational map of degree $d \geqq 2$, i.e.

$$
T(z)=\frac{a_{n} z^{n}+a_{n-1} z^{n-1}+\cdots+a_{1} z+a_{0}}{b_{m} z^{m}+b_{m-1} z^{m-1}+\cdots+b_{1} z+b_{0}},
$$

where $a_{n}, \ldots, a_{0}, b_{m}, \ldots, b_{0} \in \mathbb{C}$ (with $a_{n}, b_{m} \neq 0$ ) and $d=\max \{n, m\} \geqq 2$.

Counted according to multiplicity, every point $x \in \hat{\mathbb{C}}$ will have $d$-pre-images. If we consider the $n^{\text {th }}$ iterate $T^{n}: \hat{\mathbb{C}} \rightarrow \hat{\mathbb{C}}$ then the set of pre-images $S_{n}(x)=\{y$ : $\left.T^{n} y=x\right\}$ of a point $x \in \hat{\mathbb{C}}$ will have cardinality $d^{n}$.

Definition. The Julia set $\mathrm{J} \subset \mathbb{C}$ is defined to be the closure of the set of all periodic points $T^{n} x=x$ for which $\left|\left(T^{n}\right)^{\prime}(x)\right|>1$.

Clearly $\mathrm{J}$ is a closed $T$-invariant set and we shall be interested in the restriction $T: \mathbb{J} \rightarrow \mathbb{J}$ of the map $T$. We shall write $C^{0}(\mathbb{J})$ for the space of real valued continuous functions on $\mathbb{J}$. We denote by $\mathscr{M}_{T}$ the space of all $T$-invariant probability measures on $\mathbb{J}$.

Definition. For any continuous function $f: \mathbb{J} \rightarrow \mathbb{R}$ we can define the pressure by

$$
P(f)=\sup \left\{h(v)+\int f d v: v \in \mathscr{M}_{T}\right\},
$$

where $h(v)$ denotes the entropy of $T$ with respect to the measure $v$.

We let $h(T)=P(0)$ denote the topological entropy of $T: \mathbb{J} \rightarrow \mathbb{J}$.

The following results about entropy will be useful to us.

Lemma $1[2,4]$.

(i) $h(T)=\log d$.

(ii) The map $v \rightarrow h(v)$ is upper semi-continuous in the weak* topology.

(iii) There is a unique measure of maximal entropy $\mu_{0}$ for $T: \mathbb{J} \rightarrow \mathbb{J}$.

Statement (iii) of Lemma 1 has the following generalization.

Lemma 2 (Denker and Urbanski [1]). If $f: \mathbb{J} \rightarrow \mathbb{R}$ is a Hölder continuous function such that $P(f)>\sup f$ then $f$ has a unique equilibrium state $\mu$.

The following lemma gives us some information about the relationship between pressure and the pre-images of a point. 
Lemma 3 (Przytycki [6]). Let $x \in J$ J. Let $f$ be a Hölder continuous function on the Julia set $\mathbb{J}$ such that $P(f)>\sup f$ and let $g$ be a continuous function on $\mathbf{J}$. Then

$$
\begin{gathered}
\lim _{n \rightarrow+\infty} \frac{1}{n} \log \sum_{T^{n} y=x} e^{f^{n}(y)}=P(f) ; \\
\limsup _{n \rightarrow+\infty} \frac{1}{n} \log \sum_{T^{n} y=x} e^{f^{n}(y)+g^{n}(y)} \leqq P(f+g) .
\end{gathered}
$$

Finally, the following lemma gives us an alternative characterisation of the entropy.

\section{Lemma 4.}

(i) If $v \in \mathscr{M}_{T}$ then

$$
h(v)=\inf \left\{P(g)-\int g d v: g \in C^{0}(\mathbb{J})\right\} .
$$

(ii) If $v \in \mathscr{M}-\mathscr{M}_{T}$ then

$$
0 \geqq \inf \left\{P(g)-\int g d v: g \in C^{0}(\mathbb{J})\right\} .
$$

Proof. In fact, these two results hold for any continuous mapping $T$ of a compact metric space for which $h(T)<+\infty$ and, in the case of (i), the map $v \rightarrow h(v)$ is upper semi-continuous (cf. [10, pp. 221-222]).

\section{Proof of Theorem 1}

In this section we will give the proof of Theorem 1, using the results from the previous section. As before $f: \mathbb{J} \rightarrow \mathbb{R}$ is a Hölder continuous function satisfying $P(f)>\sup f$. We can define a map $Q: C^{0}(\mathbb{J}) \rightarrow \mathbb{R}$ by $Q(g)=P(f+g)-P(f)$. For $v \in \mathscr{M}$, we then denote the Legendre transform of $Q(g)$ by

$$
I(v)=\sup _{g \in C^{0}(\mathbb{J})}\left(\int g d v-Q(g)\right) .
$$

Given any weak $^{*}$ closed (and hence compact) subset $\mathscr{K} \subset \mathscr{M}$ we define $\rho=$ $\rho_{\mathscr{K}}:=\inf _{v \in \mathscr{K}} I(v)$.

Our proof will be based upon the following estimate.

\section{Lemma 5.}

$$
\limsup _{n \rightarrow+\infty} \frac{1}{n} \log \left(\frac{\sum_{\substack{T^{n} y=x \\ \mu_{y, n} \in \mathscr{K}}} e^{f^{n}(y)}}{\sum_{T^{n} y=x} e^{f^{n}(y)}}\right) \leqq-\rho .
$$

Proof. Fix a choice of $\varepsilon>0$. From the definition of $\rho$, for every $v \in \mathscr{K}$, there exists $g \in C^{0}(\mathbb{J})$ such that

$$
\int g d v-Q(g)>\rho-\varepsilon
$$


Thus we have that

$$
\mathscr{K} \subset \bigcup_{g \in C^{0}(\mathbb{J})}\left\{v \in \mathscr{M}: \int g d v-Q(g)>\rho-\varepsilon\right\}
$$

and by weak* compactness we can choose a finite subcover

$$
\mathscr{K} \subset \bigcup_{i=1}^{k}\left\{v \in \mathscr{M}: \int g_{i} d v-Q\left(g_{i}\right)>\rho-\varepsilon\right\} .
$$

Therefore we have the inequality

$$
\begin{aligned}
\sum_{\substack{T^{n} y=x \\
\mu_{v, n} \in \mathscr{K}}} e^{f^{n}(y)} & \leqq \sum_{i=1}^{k}\left(\sum_{\substack{T^{n} y=x \\
\frac{1}{n} g_{i}^{n}(y)-Q\left(g_{t}\right)>\rho-\varepsilon}} e^{f^{n}(y)}\right) \\
& \leqq \sum_{i=1}^{k} e^{-n\left(Q\left(g_{i}\right)+(\rho-\varepsilon)\right)}\left(\sum_{T^{n} y=x} e^{f^{n}(y)+g_{i}^{n}(y)}\right) .
\end{aligned}
$$

Taking limits we get that

$$
\begin{aligned}
& \limsup _{n \rightarrow+\infty} \frac{1}{n} \log \left(\frac{\sum \sum_{T_{y, n}^{n} \in \mathscr{K}} e^{f^{n}(y)}}{\sum_{T^{n} y=x} e^{f^{n}(y)}}\right) \\
& \leqq \sup _{1 \leqq i \leqq k}\left\{-Q\left(g_{i}\right)-\rho+\varepsilon+\limsup _{n \rightarrow+\infty} \frac{1}{n} \log \left(\sum_{T^{n} y=x} e^{f^{n}(y)+g_{i}^{n}(y)}\right)\right. \\
& \left.\quad-\liminf _{n \rightarrow+\infty} \frac{1}{n} \log \left(\sum_{T^{n} y=x} e^{f^{n}(y)}\right)\right\} \\
& \leqq \sup _{1 \leqq i \leqq k}\left\{-Q\left(g_{i}\right)-\rho+\varepsilon+P\left(f+g_{i}\right)-P(f)\right\}=-\rho+\varepsilon,
\end{aligned}
$$

where the second inequality uses Lemma 3. Since $\varepsilon>0$ can be chosen arbitrarily small this completes the proof of the lemma.

We next want to show that if $\mathscr{K}$ does not contain $\mu$ then $\rho>0$. This will follow from the next lemma.

\section{Lemma 6.}

(i) If $v \neq \mu$ then $I(v)>0$.

(ii) The map $v \rightarrow I(v)$ is lower semi-continuous on $\mathscr{M}_{T}$ and $I$ is bounded away from 0 on $\mathscr{M}-\mathscr{M}_{T}$. 
Proof. For part (i) we have that

$$
\begin{aligned}
I(v) & =\sup _{g \in C^{0}(\mathbb{J})}\left(\int g d v-P(f+g)+P(f)\right) \\
& =\sup _{g \in C^{0}(\mathbb{J})}\left(\int(g-f) d v-P(g)+P(f)\right) \\
& =\sup _{g \in C^{0}(\mathbb{J})}\left(\int g d v-P(g)\right)+P(f)-\int f d v \\
& =-\inf _{g \in C^{0}(\mathbb{J})}\left(P(g)-\int g d v\right)+P(f)-\int f d v .
\end{aligned}
$$

If $v \in \mathscr{M}_{T}$ then, by part (i) of Lemma 4, this is equal to $-h(v)+P(f)-\int f d v$, and, by the uniqueness of the equilibrium state $\mu,-h(v)+P(f)-\int f d v>0$. On the other hand, if $v \in \mathscr{M}-\mathscr{M}_{T}$, then

$$
\inf _{g \in C^{0}(\mathbb{J})}\left(P(g)-\int g d v\right)<0
$$

by part (ii) of Lemma 4 and so

$$
I(v)>0+P(f)-\int f d v \geqq P(f)-\sup f>0 .
$$

For the proof of (ii) we first notice that $I(v)=-h(v)+P(f)-\int f d v$. We then complete the proof with the lower bound in the proof of (i) above. This completes the proof of the lemma.

Since $\mathscr{K}$ is compact, we can conclude that if $\mu \notin \mathscr{K}$ then $\rho>0$. Theorem 1 now follows by setting $\mathscr{K}=\mathscr{M}-\mathscr{U}$.

Proof of Corollary 2. We shall show that for any $g \in C^{0}(\mathbb{J})$ we have that

$$
\frac{1}{\Sigma(f, n)} \sum_{T^{n} y=x} e^{f^{n}(y)} \frac{g^{n}(y)}{n} \rightarrow \int g d \mu, \quad \text { as } n \rightarrow+\infty
$$

where $\Sigma(f, n)=\sum_{T^{n} y=x} e^{f^{n}(y)}$.

Given $\varepsilon>0$, define an open neighbourhood $\mathscr{U}$ of $\mu$ by

$$
\mathscr{U}=\left\{v \in \mathscr{M}:\left|\int g d v-\int g d \mu\right|<\varepsilon\right\} .
$$

Then we may write

$$
\begin{aligned}
& \frac{1}{\Sigma(f, n)} \sum_{T^{n} y=x} e^{f^{n}(y)} \frac{g^{n}(y)}{n} \\
& =\frac{1}{\Sigma(f, n)} \sum_{\substack{T^{n} y=x \\
\mu_{y, n} \in \mathscr{U}}} e^{f^{n}(y)} \frac{g^{n}(y)}{n}+\frac{1}{\Sigma(f, n)} \sum_{\substack{T^{n} y=x \\
\mu_{y, n} \notin \mathscr{U}}} e^{f^{n}(y)} \frac{g^{n}(y)}{n} \\
& =\frac{1}{\Sigma(f, n)} \sum_{\substack{T^{n} y=x \\
\mu_{y, n} \in \mathscr{U}}} e^{f^{n}(y)}\left\{\int g d \mu+E_{n}(y)\right\}+O\left(\eta^{n}\right),
\end{aligned}
$$

where $\left|E_{n}(y)\right|<\varepsilon$ and $0<\eta<1$ is given by Theorem 1 . 
Thus we conclude (by adding appropriate constants to $g$ if necessary) that

$$
\limsup _{n \rightarrow+\infty} \frac{1}{\sum(f, n)} \sum_{T^{n} y=x} e^{f^{n}(y)} \frac{g^{n}(y)}{n} \leqq \int g d \mu+\varepsilon
$$

and

$$
\liminf _{n \rightarrow+\infty} \frac{1}{\Sigma(f, n)} \sum_{T^{n} y=x} e^{f^{n}(y)} \frac{g^{n}(y)}{n} \geqq \int g d \mu-\varepsilon .
$$

Since $\varepsilon>0$ is arbitrary, the result is proved.

\section{References}

1. Denker, M., Urbanski, M.: Ergodic theory of equilibrium states for rational maps. Nonlinearity 4, 103-134 (1991)

2. Freire, A., Lopes, A., Mañé, R.: An invariant measure for rational maps. Bol. Bras. Mat. Soc. 14, 45-62 (1983)

3. Lopes, A.: Entropy and Large Deviations. Nonlinearity 3, 527-546 (1990)

4. Lyubich, M.: Entropy properties of rational endomorphisms of the Riemann sphere. Ergodic Theory Dyn. Syst. 3, 351-385 (1983)

5. Mané, R.: On the uniqueness of the maximising measure for rational maps. Bol. Bras. Mat. Soc. 14, 27-43 (1983)

6. Przytycki, F.: On the Perron-Frobenius-Ruelle operator for rational maps on the Riemann sphere and for Hölder continuous functions. Bol. Bras. Mat. Soc. 20, 95-125 (1990)

7. Przytycki, F.: Review of "Entropy and Large Deviations" by A. Lopes. Math. Rev. 91m:58092

8. Steinmetz, N.: Rational Iteration. Berlin-New York: de Gruyter, 1993

9. Urbanski, M.: Invariant subsets of expanding mappings of the circle. Ergodic Theory Dyn. Sys. 7, 627-645 (1987)

10. Walters, P.: An Introduction to Ergodic Theory. New York-Heidelberg-Berlin: SpringerVerlag, 1982

Communicated by Ya.G. Sinai 
